\title{
Arterial peripheral disease-assessment by ankle brachial index
}

\begin{abstract}
Peripheral arterial disease is a syndrome predominantly atherosclerosis, which occurs in a partial or total occlusion of an artery, usually in the arms or legs and can be in association with other cardiovascular problems.
\end{abstract}

\begin{abstract}
Aim: It aims at the comparison of peripheral arterial disease in males and females for different age groups. This will allow correlating different risk factors with each age group. Moreover, it is in the scope of this study to learn which gender is most affected by PAD in the population living in Castelo Branco, a city in the center of Portugal.

Methods: This cross-sectional study was conducted at the Dr. Lopes Dias School of Health (ESALD), the Santa Casa da Mesericordia and the headquarters of the Volunteer Fire fighters of the city of Castelo Branco (Portugal), between September 2011 and February 2012 .

Results: There was a $17.5 \%$ prevalence of peripheral arterial disease in the city of Castelo Branco, with predominance in males $(56.9 \%)$. It was found that the occurrence of stroke, of acute myocardial infarction and the presence of hypertension increases the risk of developing pathology 7,33, 6,41 and 5,74 times, respectively.

Conclusion: This study reveals the importance of high prevalence of peripheral arterial disease in patients with more than 75 years old $(83.3 \%)$ and alert for the presence of cardiovascular problems which increases the occurrence of this disease in about eight times. Further investigations are needed to properly assess the importance of the risk factors for the various age groups and verify the impact of preventive and therapeutic measures on the complications of this disease overlooked by society.
\end{abstract}

Keywords: peripheral arterial disease, ankle-brachial index, cardiovascular risk factors
Volume I Issue 4 - 2014

\author{
Cristiana Ramalhao, Ana Alves, Alexandre \\ Pereira \\ Department of Cardiology, Royal Bournemouth Hospital, UK
}

Correspondence: Cristiana Ramalhao, Cardiac Department, Royal Bournemouth Hospital, BH7 7DW, Dorset, United Kingdom, Tel 44-7463-804-186,

Email cristiana_ramalhao@hotmail.com

Received:June 13,2014 | Published: July 29, 2014

\section{Introduction}

The peripheral arterial disease (PAD) is a pathological entity related with atherosclerotic and thromboembolic processes affecting the aorta, visceral branches and the arteries of the lower limbs. ${ }^{1-5}$ It is caused in most of cases by atherosclerosis, which leads to the development of stenosis and occlusions in the major arteries of the lower limbs movement, with the arteries of the upper limbs more rarely affected. ${ }^{1,5,6}$ The main physiological disorder is the limitation of arterial blood flow, leading to ischemia of tissues irrigated by the affected artery, hence the signs and symptoms of the disease. The most common symptom of PAD is intermittent claudication (IC), whose prevalence is about $3 \%$ in patients with 40 years old, rising to $6 \%$ inpatients over 60 year old. ${ }^{4,5}$

Identification of risk factors is extremely important for the early diagnosis and treatment of PAD, as this is associated with reduced functional capacity and quality of life. Moreover, it has been related to an increase in cardiovascular morbidity and mortality, especially after acute myocardial infarction and/or stroke. ${ }^{1,5,7,8}$ Basic clinical examination is usually based on symptoms such as IC, pain at rest and abnormal signs such as decreased peripheral pulses, ischemic ulceration and gangrene. PAD can be diagnosed in most cases by measuring the ankle-brachial index. ${ }^{4,7}$. The present study aims at comparing PAD in males and females for different age groups. This will allow correlating different risk factors with each age group. Moreover, it is in the scope of this study to learn which gender is most affected by PAD in the population living in Caste lo Branco, a city in the center of Portugal.

\section{Materials and methods}

\section{Methodology}

This cross-sectional study was conducted at the Dr. Lopes Dias School of Health (ESALD), the Santa Casa da Misericordia and the head quarters of the Volunteer Fire fighters of the city of Castelo Branco (Portugal), between September 2011 and February 2012. Inclusion criteria were predefined as age $\geq 20$ year old and being a citizen of Castelo Branco. Upon explaining the exam to the volunteer and the consent form signed, subjects were asked to complete a questionnaire to obtain information such as demographic data, symptoms of PAD, cardiovascular events, and existence of risk factors. The included study variables were: age; gender; Body Mass Index (BMI) class, defined according to the World Health Organization (WHO). ${ }^{9}$ smoking habits; dyslipidaemia and cardiovascular events sstroke, AMI and Transient ischemic accident (TIA) $\}$, family history of cardiovascular conditions (CVP), hypertension, diabetes mellitus (DM), IC (limiting and non-limiting) and discoloration of the skin. The IC stages in the lower limb were adapted from the Fontaine classification in stage-I asymptomatic; stage-IIa non-limiting IC; stage-IIb limiting IC; stageIII pain at rest and stage-IV necrotic lesions, ulcers or gangrene. ${ }^{10}$ The presence of hypertension was considered if: systolic pressure $\geq 140 \mathrm{~mm} \mathrm{Hg}$ and/or diastolic pressure $\geq 90 \mathrm{~mm} \mathrm{Hg}$, or controlled by anti hypertensive. ${ }^{11}$ 
The ABI was measured using a Nicolet TM Vascular IMEXDOPCT+TM (a flow measurement device), with an $8 \mathrm{MHz}$ probe. The patient remained supine for at least 5 minutes before the palpation of the radial artery pulses of both arms, in order to identify the pulse asymmetry. If this was verified, the na measurement of blood pressure (BP) in both arms was performed, otherwise a measurement was taken in only one artery, preferably the left brachial. In the lower limbs, the dorsal artery of the foot and posterior tibial artery were evaluated. The ABI was obtained by calculating the ratio between the smallest value of systolic pressure of each leg and arm, determined for each extremity. It was considered the subject had PAD when $\mathrm{ABI} \leq 0.90$, and had a non-compressible artery when $\mathrm{ABI} \geq 1.40$. Within this range, the $\mathrm{ABI}$ was classified as normal for values between 1.0 and
1.39; border line between 0.91 and 0.99 ; mild to moderate ischemia between 0.41 and 0.90 and severe ischemia when $\mathrm{ABI}<0.40 .{ }^{10}$

\section{Sample characterization}

873 individuals, predominantly female (55\%), aged between 20 and 99 years (mean age of $56 \pm 21.9$ years) were included in the study. The sample population is characterized in Table 1, for which a higher prevalence of risk factors was seen in females, with the exception of CVP. Regarding smoking and the presence of hypertension, differences between the sexes are not statistically significant. Regarding the division of BMI for the different classes, it was found that there was a high prevalence of overweight individuals (33.6\%), regardless of the gender (Table 1).

Table I Study sample characteristics.

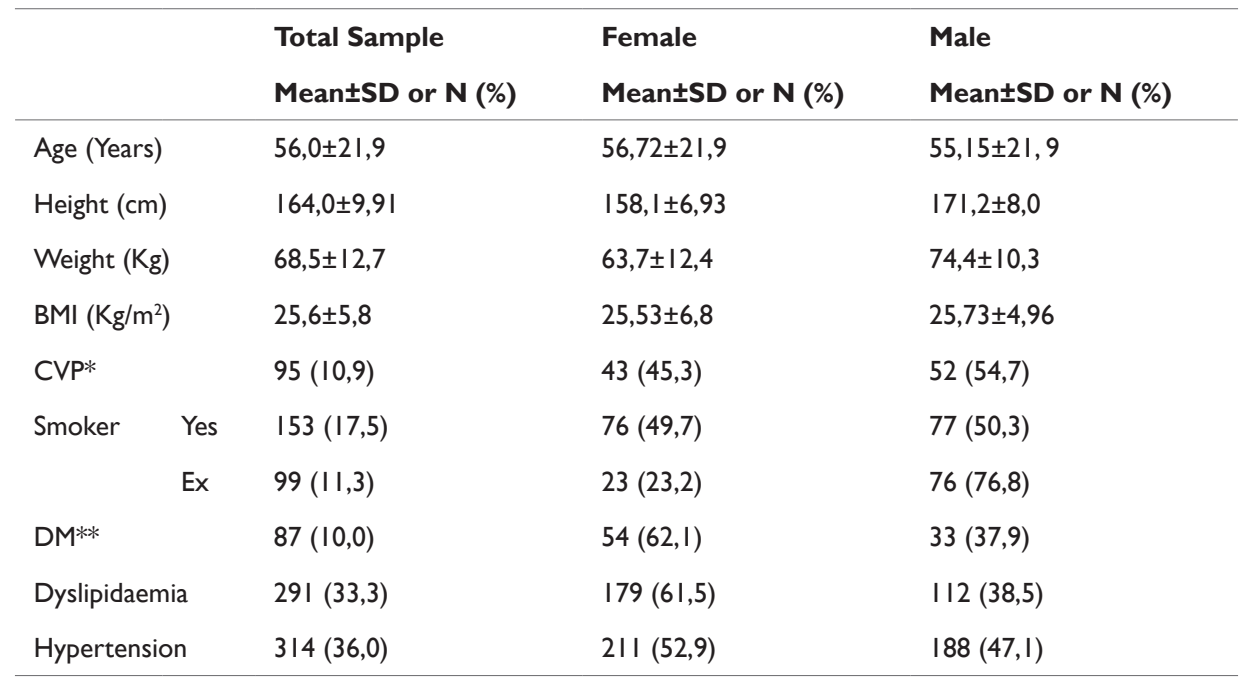

*CVP, cardiovascular problems; **DM, diabetes mellitus-2

\section{Statistical analysis}

Statistical analysis was performed with the Statistical Package for Social Sciences (SPSS ${ }^{\circledR}$ ) version 20. Simple descriptive analysis was used, where values are presented as mean \pm standard deviation (SD) for continuous variables, and total (n) and percentage (\%) for discrete variables. To test the hypothesis, the chi-square test of independence was used. In order to verify the influence of risk factors on the probability of developing PAD, univariate and multivariate logistic regression models were adjusted, as described by Moroco. ${ }^{12}$ Statistical significance was established at $\mathrm{p}<0.05$.

\section{Results}

Amongst the 873 subjects that participated in the study, 153 $(17.5 \%)$ had an $\mathrm{ABI}<0.90$. There was a mean $\mathrm{ABI}$ of $1.07 \pm 0.19$ for the right leg with minimum and maximum values of 0.23 and 2.17, respectively. For the left leg, it was found a mean ABI of $1.06 \pm 0.17$, with a minimum and maximum value of 0.28 and 2.17 , respectively. In analyzing the results for the different classes of $\mathrm{ABI}$ for each leg (Graph 1\&2), it was found that a normal ABI was the most prevalent for both legs. However, when evaluating the presence of pathology, moderate to mild ischemia is the most frequent ABI class, with a percentage of $13.3 \%$ (left leg) and $12.3 \%$ (right leg). It is also important to highlight the increased prevalence of an $\mathrm{ABI}>1.40$, which corresponds to a non-compressible artery.

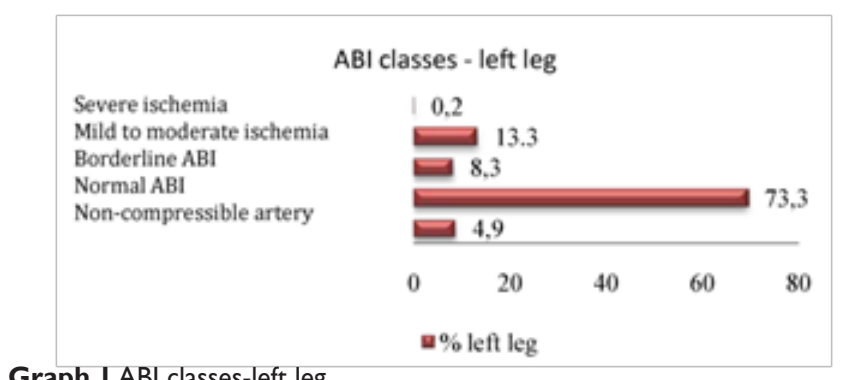

Graph I ABI classes-left leg.

*ABI, ankle brachial index

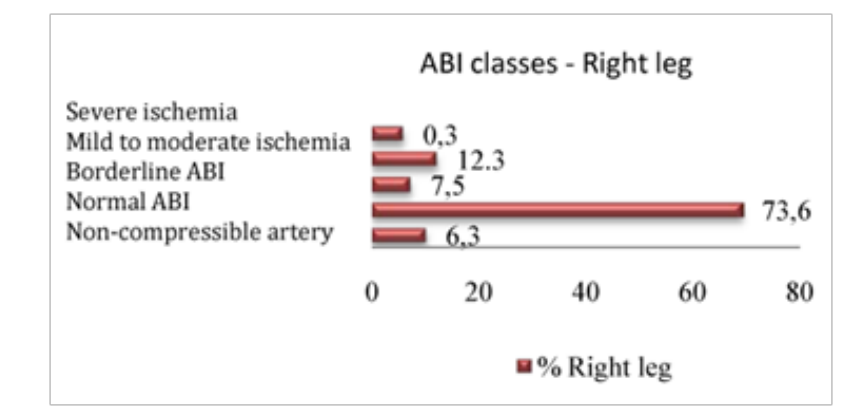

Graph 2 ABI classes-right leg.

*ABI, ankle-brachial index 
Severe ischemia is present only in individuals aged over 85 years. In terms of the $\mathrm{ABI}$ correspondent to a non-compressible artery, despite its presence throughout all age groups, it was observed to have increased more than the double for subjects above 75 yeras, compared with their younger counter parts. In the sample the study comprised, it was found a prevalence of PAD of $17.5 \%$, mainly amongst males $(56.9 \%)$. Graph 3 shows the analysis of the PAD prevalence distributed by gender and age, wherein this increase for older age groups in both genders can be observed. For the age groups with 55-64year and 65-74year it was found a greater PAD prevalence in males than in females; however, this relationship appears to be reversed for patients older than 75years, with a higher prevalence in the female gender class (Graph 3). Studying the patients with PAD, it was found that $28.1 \%$ of those reported pain at rest and $27.5 \%$ anon-limiting IC, the two most common conditions observed in this study. Graph 4 represents the Leriche-Fontaine classification for these patients with PAD. From here, it was apparent a difference in terms of gender: where as the pain at rest was seen more frequently in females $(20.9 \%)$, non-limiting IC had a higher frequency in males (15.7\%) (Graph 4). Limiting IC was more frequent in females $(9.2 \%)$ compared with males $(5.2 \%)$. This also emphasizes the importance of detection of symptomatic individuals with significant prevalence (22.9\%). Univariate logistic regression models were performed, by using PAD as the dependent variable, and pain with exercise, pain at rest, IC, cold extremities and changes in skin color as independent variables. This aimed at studying the relationship between PAD and symptoms, as shown in Graph 5.

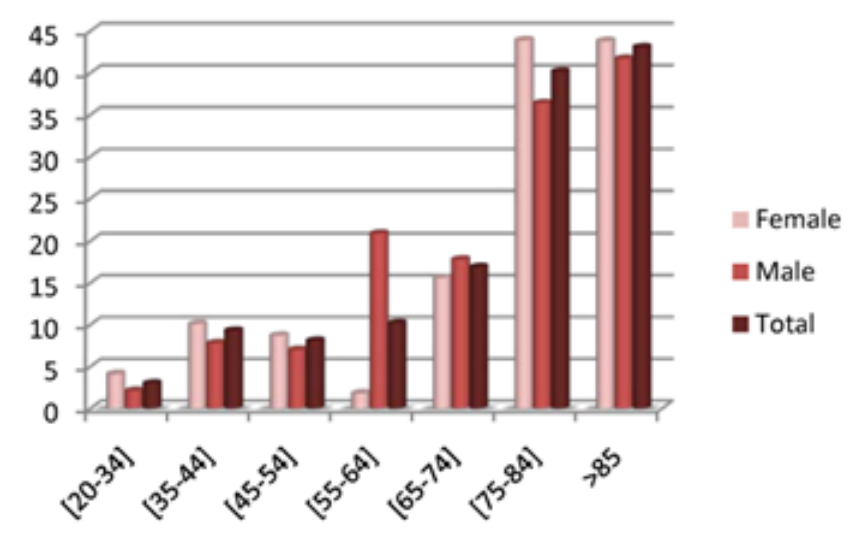

Graph 3 PAD prevalence by age group.

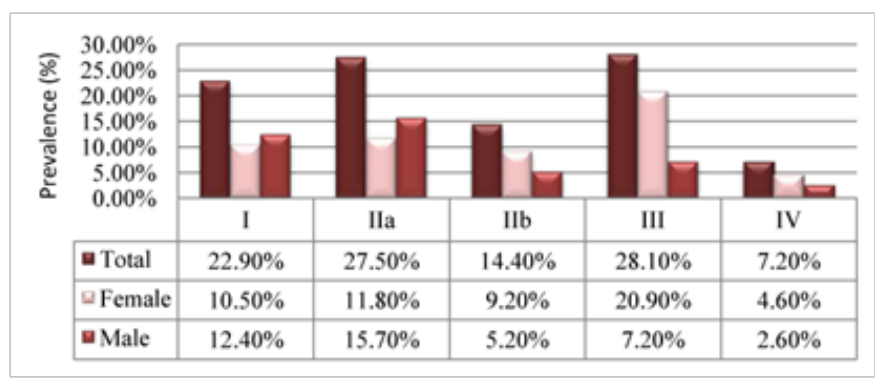

Graph 4 Leriche-Fontaine classifications.

I, asymptomatic; Ila, non-limiting IC; Ilb, limiting IC; III, pain at rest; IV, necrotic lesions

Regarding the symptoms, it appears that resting pain is the symptom less associated with the probability of PAD (OR=1.15).
Moreover, patients who have pain with exercise are approximately 2 times more likely to have PAD. Referring to risk factors for PAD, it was found that, in absolute terms, the most prevalent risk factor was hypertension (78.4\%), followed by dyslipidaemia (45.1\%) and the presence of back ground CVP (33.3\%). Split by gender, the most prevalent risk factor is still hypertension, with $42.5 \%$ in females' and $35.9 \%$ in males. It is also relevant to highlight a significant statistical difference between genders for the presence of DM, with $15.0 \%$ in females and $5.9 \%$ in males. The same is true for the CVP history, affecting males 1.6 times more than females. In regards to smoking habits, active smokers comprised the fourth largest percentage of individuals with PAD (27.5\%); however, there was no statistically significant evidence for its influence on PAD $(\rho=0.74)$. Graph 6 shows the univariate logistic regression analysis of the association of risk factors with PAD. Considering these, gender $(\rho=0.60)$, history of TIA $(\rho=0.80)$, family history of cardiovascular conditions $(\rho=0.60)$ and smoking $(\rho=0.16)$ did not have a predictive effect on PAD (Graph 6).

\begin{tabular}{|rrrl} 
Pain with exercise & 1.63 & CI 95\% & P value \\
Pain at rest & 1.15 & $1,44-1,85$ & 0,000 \\
IC & 1.53 & $1,01-1,32$ & 0,031 \\
skin color changes & 1.59 & $1,00-2,34$ & 0,040 \\
cold extremities & 1.36 & $1,36-1,87$ & 0,000 \\
& & $1,21-1,54$ & 0,000 \\
m Odds Ratio & & &
\end{tabular}

Graph 5 Odds Ratio-Symptomatology-PAD.

*IC, intermittent claudication; $\mathrm{Cl}$, confidence interval

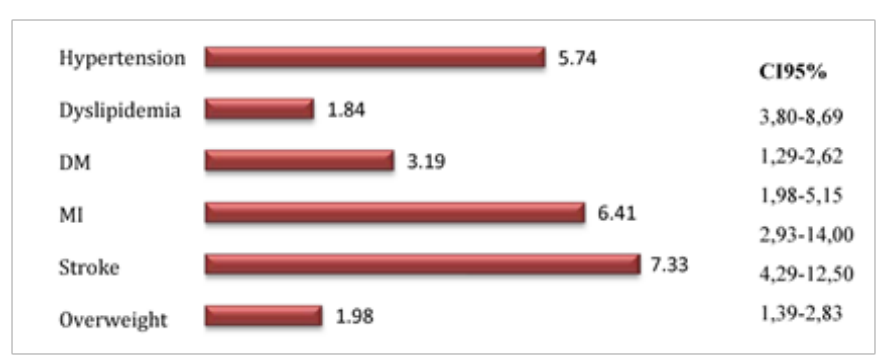

Graph 6 Odds Ratio-Risk factors PAD.

*DM, diabetes mellitus; MI, myocardial infarction

It is noteworthy that the occurrence of stroke, MI and hypertension increase the risk of developing PAD by $7.33,6.41$ and 5.74 times, respectively. A multivariate logistic regression model was adjusted to the data points in order to ascertain whether the risk factors remain predictive for the risk of PAD. It was found that age, history of stroke, MI, presence of DM and hypertension all contributed independently to an increased risk of PAD.

\section{Discussion}

The ABI is a measurement tool that provides objective information, so it is considered the gold standard technique for the diagnosis and evaluation of therapeutic efficacy in treating PAD. This test has a sensitivity of $79 \%$ and a specificity of $96 \% .{ }^{10}$ Analyzing the prevalence of the disease, the observed results in this study $(17.5 \%)$ were similar to those reported in literature (estimated at $10-25 \%{ }^{13,14}$ Hirsh et al. ${ }^{5}$ showed that PAD is easily detectable and diagnosed by an $\mathrm{ABI}<0.90$ in the primary diagnosis with a prevalence of $29 \%$ ). 
However, a study of prevalence of PAD performed by the Portuguese Society of Angiology and Vascular Surgery in 2008, using the ABI, revealed a prevalence of $5.9 \% .^{14}$ This value was lower than that described in literature and the results herein reported, which can be explained by the large sample size $(n=5731)$ and higher diversity of the characteristics analyzed. There is, none the less, a strong association between advanced age ( $\geq 70$ years) and the prevalence of PAD across all studies. In the NHANES report, it was noted that the prevalence of PAD was $4.3 \%$ in individuals aged over 40year, compared with $14.5 \%$ for those aged 70 years or over. ${ }^{15}$ Criqui et al. ${ }^{16}$ reported that the prevalence of PAD is $2-3 \%$ in individuals less than 50 years, compared with $20 \%$ in those over 75 years of age. Even more relevant results were observed by the Cardiovascular Health Study. A larger number of older people was recruited as part of that study, during which it was found a prevalence of PAD in $25 \%$ of people aged $80-84$ year that increased to $30 \%$ in those over 85 years. ${ }^{17}$ Similarly, the current study observed a significant increase in the prevalence of PAD in older individuals: younger age groups presented a PAD of $47.5 \%$, which increased to $83.3 \%$ in those aged over $75 y$ ears of age the prevalence of PAD is, this number decreases to $47.5 \%$ in younger

McDermott et al. ${ }^{21}$ showed that PAD is more prevalent in men $(59.2 \%)$ than in women $(40.8 \%)$. Another study, by Baena-Diez et al. ${ }^{19}$ has also found a prevalence of PAD more pronounced in men $(55.4 \%)$ compared with that in women (44.6\%). In the same line, Bozkurt et al. ${ }^{18}$ also concluded that the prevalence of PAD is higher in males $(52.8 \%)$ compared to females (47.2\%). In contrast, Meijer et al. ${ }^{22}$ showed in the Rotterdam study, composed of a sample of 7715 individuals, that the prevalence of PAD in women $(20.5 \%)$ was larger than that in men $(16.9 \%)$. The current study has identified that, although not statistically significant, a prevalence of this pathology in ages over 75 years was more pronounced in women $(87 \%)$ than in men $(78.1 \%)$. However, for ages between 55 and 74 years this relationship is reversed, with a higher prevalence in men $(38.7 \%)$ compared to $17.4 \%$ in women The identified risk factors for this disease still remain controversial. However, there is experimental evidence that highlights the influence of age, hypertension, dyslipidaemia and DM..$^{13,18-20,22}$ In regards to smoking habits, some authors argue this to be the most important risk factor for the development of complications of PAD, with active smokers presenting an increased risk of developing PAD by four times and an earlier onset of symptoms by about ten years. ${ }^{23}$ According to Brandao et al. ${ }^{20}$ smokers with PAD have lower survival rates when compared to non-smokers, with death attributed to a major vascular event. The former also have a higher probability of progression to critical limb ischemia, as well as a risk of amputation twice as higher. In this study it was not possible to verify the impact of smoking habits in patients with PAD, as no significant correlation was found for the presence of the disease. These can be justified by the low percentage of active smokers in the sample (17.5\%), with 153 smokers in a total of 873 participants. According to literature, DM was found to increase the risk of developing PAD by four-fold. It was also associated with an increased risk of premature cardiovascular events and mortality amongst patients already diagnosed with PAD. ${ }^{8}$ In the NHANES report it was found that $26 \%$ of individuals with this condition had DM. ${ }^{15}$ The Multi-Ethnic Study of Atherosclerosis (MESA) found that $26 \%$ of women and $27.5 \%$ of men with $\mathrm{ABI}<0.90$ had DM. ${ }^{8}$ In the current study, the presence of DM was found to be statistically significant for the presence of PAD, with $15.0 \%$ in females and $5.9 \%$ in males. It was also found that patients with DM have an increased risk of developing PAD by 3.19 times.
In the Framingham Heart Study, high cholesterol levels were associated with an increased risk in developing IC. ${ }^{24}$ The NHANES report found that over $60 \%$ of individuals with PAD had hypercholesterolemia, ${ }^{15}$ whilst the PARTNERS program reported that the prevalence of hyperlipidemia in patients with PAD was $77 \% .{ }^{25}$ The current study has corroborated these results, having found a prevalence of high cholesterol levels in $45.1 \%$ of individuals with PAD. There also appears to be increased risks of developing PAD by 1.84 times in individuals with hyperlipidemia, when compared to those with regular cholesterol levels. Most epidemiological studies have shown a strong association between hypertension and PAD, observed in between 50\% to $92 \%$ of patients with the condition. ${ }^{15,25-27}$ In the NHANES report and the PARTNERS Program, ${ }^{5}$ PAD and hypertension were observed in $74 \%$ and $92 \%$ of participants, respectively. ${ }^{25,27}$ The Cardiovascular Health Study reported that $52 \%$ of patients with an $\mathrm{ABI}<0.90$ showed hypertension, ${ }^{17}$ whilst the Framingham Study stated that in individuals with hypertension there is an increased risk to develop IC by 2.5 to 4 times. ${ }^{28}$ The current study is concordant with the literature, showing a prevalence of hypertension in $78.5 \%$ of patients with PAD, although more frequent in females ( $42.5 \%$ of the cases). It was also found that hypertensive individuals have an increased risk of developing PAD 5.7 times higher than individuals without PAD.

The Seventh Report of the National Committee of the United States of America on Prevention, Detection, Evaluation, and Treatment of High Blood Pressure (SHEP) acknowledged that PAD is equivalent in terms of risk to ischemic heart disease. ${ }^{3}$ Patients with hypertension and PAD have a high risk of stroke and MI, independent of the presence of other risk factors. ${ }^{25,27}$ In the SHEP study, elderly patients with hypertension and an $\mathrm{ABI} \leq 0.90$ were found to have two to three times' higher risk of cardiovascular mortality when compared to the other participants. ${ }^{3}$ It is worth of note the limitations of the current study, e.g. there presentativeness of the questionnaires in the sample population. The IC was evaluated through this method and not by objective examination, as it would be expected. However, these types of enquiries are important to identify possible subjects with the disease, hence ensuring the prevention and treatment of the condition more quickly and efficiently. Another limitation in this study relates to the diagnosis of some risk factors, such as dyslipidaemia and DM. These were obtained through information given by the participants, and not supported by diagnostic procedures. In regards to the group of smokers, and as already identified, the low sample representatively made it impossible to demonstrate significant differences in smokers with PAD and without PAD. Finally, this data does not allow defining the degree to which other conditions may affect the symptoms and physical appearance of the lower limbs.

\section{Conclusion}

The chances for an early diagnosis of PAD are increased by recurring to specific tests aimed at detecting indirect signals, as many patients are asymptomatic but still carry PAD. ${ }^{13,20}$ Several epidemiological studies have shown that men aged above 60 years have a very high prevalence of atherosclerosis complications. ${ }^{17,25,26}$ These findings allow ascertaining that the risk factors for atherosclerosis and its corresponding complications have therefore different effects in both men and women. Thus, more careful studies into these differences are very important so that the most appropriate preventive and therapeutic measures can be taken for each case. ${ }^{25}$ Literature data also suggests that the hierarchical importance of risk factors may change with age. Hence, studies are needed to control the disease in younger age 
groups. ${ }^{4,18,29}$ Still, there are no studies that demonstrate a relationship between the most prevalent risk factors for each age group and gender. Considering the diagnostic means of DAP are nowadays easy to put in practice, for a disease with such great impact into the quality of life, it is suggested that more effort be put into better understanding this over looked pathology in clinical practice.

\section{Acknowledgments}

None.

\section{Conflicts of interest}

Authors declare that there is no conflict of interest.

\section{References}

1. Francisco Silva HS, Figueiredo J, Conde J. Doenca arterial periferica no Idoso. Revista Cardio pulmonar. 2011;4:12.

2. Dieter RS, Chu WW, Pacanowski JP, et al. The significance of lower extremity peripheral arterial disease. Clin Cardiol. 2002;25(1):3-10.

3. Chobanian AV, Bakris GL, Black HR, et al. The seventh report of the joint national committee on prevention, detection, evaluation, and treatment of high blood pressure:the JNC 7 report. JAMA. 2003;289(19):2560-2572.

4. Mahe G, Le Faucheur A, Noury-Desvaux B. Ankle-brachial index and peripheral arterial disease. $N$ Engl J Med. 2010;2(5):470-471.

5. Hirsch AT, Criqui MH, Treat-Jacobson D, et al. Peripheral arterial disease detection, awareness, and treatment in primary care. JAMA. 2001;286(11):1317-1324.

6. McDermott MM, Greenland P, Liu K, et al. Leg symptoms in peripheral arterial disease: associated clinical characteristics and functiona impairment. JAM. 2001;286(13):1599-1606.

7. Fowkes FG, Housley E, Riemersma RA, et al. Smoking, lipids, glucose intolerance, and blood pressure as risk factors for peripheral atherosclerosis compared with ischemic heart disease in the Edinburgh Artery Study. Am J Epidemio. 1992;135(4):331-340.

8. McDermott MM, Liu K, Criqui MH, et al. Ankle-brachial index and subclinical cardiac and carotid disease:the multi-ethnic study of atherosclerosis. Am J Epidemiol. 2005;162(1):33-41.

9. Formiguera X, Canton A. Obesety:epidemiology and clinical aspects. Best Pract Res Clin Gastroentero. 2004;1 18(6):1125-1146.

10. Rooke TW, Hirsch AT, Misra S, et al. 2011 ACCF/AHA focused update of the guideline for the management of patients with peripheral artery disease (updating the 2005 guideline):a report of the American College of Cardiology Foundation/American Heart Association Task Force on practice guidelines. J Am Coll Cardiol. 2011;58(19):2020-2045.

11. Mancia G, De Backer G, Dominiczak A, et al. Guidelines for the management of arterial hypertension: the task force for the management of arterial hypertension of the European Society of Hypertension $(\mathrm{ESH})$ and of the European Society of Cardiology (ESC). Eur Heart J. 2007;28(12):1462-536.

12. Maroco J. Analise estatistica com utilizacao de SPSS. 3rd edition. Lisboa: 2007.

13. Bartholomew JR, Olin JW. Pathophysiology of peripheral arterial disease and risk factors for its development. Cleve Clin J Med. 2006;73(Suppl 4):S8-S14.
14. Ferreira M, Barroso P, Duarte N. Peripheral arterial disease. Revista Portuguesa de Clinica Geral. 2010;26(5):502-509.

15. O'Hare AM, Glidden DV, Fox CS, et al. High prevalence of peripheral arterial disease in persons with renal insufficiency: results from the National Health and Nutrition Examination Survey 1999-2000. Circulation. 2004;109(3):320-323.

16. Criqui $\mathrm{MH}$, Vargas V, Denenberg JO, et al. Ethnicity and peripheral arterial disease:the San Diego Population Study. Circulation. 2005;112(17):2703-2707.

17. Newman AB, Siscovick DS, Manolio TA, et al. Ankle-arm index as a marker of atherosclerosis in the Cardiovascular Health Study. Cardiovascular Heart Study (CHS) Collaborative Research Group. Circulation. 1933;88(3):837-845.

18. Bozkurt AK, Tasci I, Tabak O, et al. Peripheral artery disease assessed by ankle-brachial index in patients with established cardiovascular disease or at least one risk factor for atherothrombosis-CAREFUL study:a national, multi-center, cross-sectional observational study. BMC Cardiovasc Disord. 2011;11:4.

19. Baena-Diez JM, Alzamora MT, Fores R, et al. Ankle-brachial index improves the classification of cardiovascular risk:PERART/ARTPER Study. Rev Esp Cardiol. 2011;64(3):186-192.

20. Brandao AC, Trindade DM, Pinhel MA, et al. Avaliacao do perfil lipidico na doenca arterial periferica. J Vasc Br. 2005;4(2):129-136.

21. McDermott MM, Liu K, Greenland P, et al. Functional decline in peripheral arterial disease:associations with the ankle brachial index and leg symptoms. JAMA. 2004;292(4):453-461.

22. Meijer WT, Grobbee DE, Hunink MG, et al. Determinants of peripheral arterial disease in the elderly: the Rotterdam study. Arch Intern Med. 2000;160(19):2934-2938.

23. Norgren L, Hiatt WR, Dormandy JA, et al. Inter-Society Consensus for the Management of Peripheral Arterial Disease (TASC II). Eur J Vasc Endovasc Surg. 2007;33(Suppl 1):S1-S75.

24. Kannel WB, McGee DL. Update on some epidemiologic features of intermittent claudication:the Framingham Study. $J$ Am Geriatr Soc. 1985;33(1):13-18.

25. Selvin E, Erlinger TP. Prevalence of and risk factors for peripheral arterial disease in the United States:results from the National Health and Nutrition Examination Survey, 1999-2000. Circulation. 2004;110(6):738-743.

26. Wattanakit K, Folsom AR, Selvin E, et al. Risk factors for peripheral arterial disease incidence in persons with diabetes:the Atherosclerosis Risk in Communities (ARIC) Study. Atherosclerosis. 2005;180(2):389397.

27. Olin JW. Hypertension and peripheral arterial disease. Vasc Med. 2005;10(3):241-246.

28. Murabito JM, D'Agostino RB, Silbershatz H, et al. Intermittent claudication. A risk profile from The Framingham Heart Study. Circulation. 1977; 96(1):44-49.

29. Neto SS, Nascimento JLM. Peripheral vascular disease: new perspectives of risk factors. Rev Para Med.2007;21(2):35-39. 